\title{
Bladder dysfunction after acute urinary retention in the rats: a novel over active bladder model
}

\author{
Motoaki Saito*, Shogo Shimizu, Yukako Kinoshita, Itaru Satoh, Kohei \\ Shomori, Fotios Dimitriadis and Keisuke Satoh
}

Department of Pathophysiological and Therapeutic Science, Division of Molecular Pharmacology, Tottori University Faculty of Medicine, 86 Nishi-machi, Yonago, 683-8503 Tottori, Japan

Keywords: acute urinary retention, over active bladder (OAB), rat

Correspondence:

Motoaki Saito, MD, PhD

Department of Pathophysiological and Therapeutic Science, Division of Molecular Pharmacology, Tottori University Faculty of Medicine, 86 Nishi-machi, Yonago, 683-8503 Tottori, Japan

$\begin{array}{ll}\text { Telephone: } & +81-859-38-6162 \\ \text { FAX: } & +81-859-38-6160 \\ \text { e-mail address: } & \text { saitomo@.med.tottori-u.ac.jp }\end{array}$




\section{Abstract}

As there are increasing evidence that benign prostatic hyperplasia and its related acute urinary retention (AUR) induce over active bladder (OAB) syndrome, we investigated the effects of AUR on bladder function over a four-week period in a rat model. Ten-week-old female Sprague Dawley rats were used in this study. AUR was induced by clamping the distal urethra of each rat with a small clip, and then infusing 3 $\mathrm{ml}(0.6 \mathrm{ml} / \mathrm{min})$ of saline with an infusion pump through a transurethral catheter (22G). The obstruction was sustained for 60 minutes and the clip was removed and then the bladder was allowed to drain through the catheter. The bladder function was estimated by voiding behavior studies (at 3 days, 1, 2, 3 and 4 weeks), cystometric studies (at 2 and 4 weeks) and organ bath studies using $\mathrm{KCl}$ and carbachol (at 2 and 4 weeks). Furthermore, we evaluated histological changes in the rat bladder 2 and 4 weeks after the induction of AUR. The same parameters were also measured in non-AUR rats (control group). The rat bladder weight in the AUR group at two weeks was significantly larger than that of the controls, and returned to the control level 4 weeks after the AUR episode. The voiding behavior studies showed significant increase in micturition frequency per day and decrease in single voiding volume 3 days after the induction of AUR, and this voiding behavior was continued for more than two weeks. The cystometric studies showed a significant decrease in single-voided volume at 2 weeks rat. However, no significant changes of the other parameters were observed in the rats. The histological studies showed significant infiltration of neutrophils and lymphocytes, as well as increase in turnover of epithelium in AUR rats at 2 weeks, while significant increases in fibrosis in submucosal layer were observed in AUR rats at 4 weeks. This study demonstrated that bladder dysfunction in the rat model caused by 
AUR needs more than two weeks of recovery period. The AUR-associated alterations in the bladder may represent a key clue to understand the underlying pathophysiological mechanisms, which take place in OAB syndrome. 


\section{Introduction}

Acute urinary retention (AUR) is a significant public health issue with significant consequences to the affected individual. AUR results in prostatectomy in 24-42\% of men presenting in outpatient clinics and emergency rooms in Britain and North America [1]. Traditionally, prolonged bladder overdistension has been thought to produce stretch damage of the detrusor muscle and other bladder microstructures, resulting in reduced contractility of the detrusor muscle. Several reports have indicated that the biochemical and metabolic status of the bladder is altered after prolonged overdistension [2-5]. In previous reports in our laboratory we have demonstrated that ischemia-reperfusion injury (I-RI) might be at least partly responsible for the prolonged bladder dysfunction occurring after AUR $[5,6]$. In the above studies we showed that AUR induces a reduction in blood flow to bladder tissue resulting in partial ischemia and hypoxia; after subsequent catheterization, the blood supply recovers, allowing reperfusion [5, 6]. As with I-RI of other organs, ischemia followed by subsequent reperfusion in the bladder leads to oxidative stress, inflammation, neutrophil infiltration, which might contribute to the delayed recovery of bladder function [7]. Recently, we reported that edaravone, a radical scavenger, reduced the oxidative stress and prevented the bladder dysfunction caused by AUR and subsequent catheterization [6]. However, there is only limited information available about bladder dysfunction caused by AUR; which are the pathogenetic mechanisms of the dysfunction of the bladder, is the bladder dysfunction reversible and for how long does this dysfunction continue? In order to clarify these questions, we investigated the effects of AUR on bladder function over a four-week period in a rat model. 


\section{Materials and methods}

All animal experiments were performed in accordance with the guidelines set by the Tottori University Committee for Animal Experimentation. Ten-week-old female Sprague-Dawley rats (n=12; AUR group) weighing 230-270 g (SLC, Shizuoka, Japan) were anaesthetized with sodium pentobarbital (50 mg/kg, i.p.). AUR was induced by clamping the distal urethra of each rat with a small clip, and then infusing $3 \mathrm{ml}(0.6$ $\mathrm{ml} / \mathrm{min}$ ) of saline with an infusion pump through a transurethral catheter (22G). The obstruction was sustained for 60 minutes and then bladder was allowed to drain through the catheter. The bladder function was estimated by voiding behavior studies, cystometric studies and organ bath studies. Furthermore, we evaluated histological changes in the rat bladder 2 and 4 weeks after the induction of AUR. The same parameters were also measured in the non-AUR rats $(n=6$; control group). After cystometric studies, the rats were sacrificed with an overdose of pentobarbital (60 $\mathrm{mg} / \mathrm{kg}$, i.p.). The rat bladder was removed and a sample of it was immediately processed for functional and histological studies.

The voiding behavior studies at 3 days, 1, 2, 3 and 4 weeks of AUR rats were performed according to methods used in our previous studies [8, 9]. All rats received food and water ad libitum. The micturition parameters which have been registered included micturition reflex obtained were micturition frequency, total urine output, and single voided volume.

The cystometric studies at 2 or 4 weeks of AUR rats were performed according to methods used in our previous studies [8, 9]. Cystometry was performed under 
urethane anesthesia (1.0 g/kg, subcutaneously). In short, each rat's abdomen was opened using a lower midline incision and the bladder was exposed. Cystometry was carried out with a $22 \mathrm{G}$ catheter inserted into the apex of the bladder dome in order to record the bladder pressure and to fill the bladder with physiological saline $(0.9 \%$ $\mathrm{NaCl}$. External bladder filling was carried out using an infusion pump (5200, TOP, Tokyo) at a constant rate of $12 \mathrm{ml} / \mathrm{hr}$ until micturition was detected. A cystometry catheter was connected to an external pressure transducer (P2310, Gould, Eastlake, $\mathrm{OH}$ ) for the measurement of the intravesical pressure. The intravesical pressure was recorded on a personal computer (Macintosh G3, Apple Computer) via a bridge amplifier (San-ei, Tokyo, Japan) and a multiport controller (PowerLab/16sp, AD Instruments). The following parameters were evaluated: bladder capacity, maximum detrusor pressure during voiding, single-voided volume, and residual urine volume. The maximum detrusor pressure was defined as the instantaneous pressure minus the post-contraction resting pressure. In each animal, approximately 10-12 voiding cycles were recorded and then the mean of the voiding cycles was calculated.

The functional studies were conducted according to methods used in our previous reports $[6,8,9]$. The rat bladder dome was immediately removed and separated from the bladder base at the level of the ureteral orifices. Razor blades were used to obtain uniform longitudinal strips of the posterior wall of the bladder dome (1.5 x $5 \mathrm{~mm})$. One end of the bladder strip was fixed to a hook in the bottom of the muscle chamber, and the other end was fastened to a force displacement transducer. Muscle strips were mounted in organ baths (25 ml) containing Krebs-Henseleit solution, and bubbled with 
5\% $\mathrm{CO}_{2}$ and $95 \% \mathrm{O}_{2}(37 \mathrm{C})$. The upper hook was suspended from a transducer (type 45196A, San-ei Instruments, Tokyo, Japan), and the lower hook was fixed to a plastic support leg attached to a micrometer (Mitutoyo, Tokyo, Japan). Each strip was equilibrated unstretched for 30 minutes. A load of $1.0 \mathrm{~g}$ was applied to each strip by micrometer adjustment, and the load was readjusted to this level 30 minutes later. Changes in the tone of the strips were measured isometrically by means of force transducers, and the data were recorded on a personal computer (Macintosh G3, Apple Computer, Cupertino, CA) with the use of Chart v 3.6.9 software and a PowerLab/16sp data acquisition system (ADInstruments, Castle Hill, Australia). Cumulative concentration-response curves to carbachol and $\mathrm{KCl}(100 \mathrm{mM})$ were constructed.

After each bladder was transected at the level of the ureteral orifice, the bladder dome was immediately fixed with $10 \%$ formalin. After fixation, the tissues were embedded in paraffin. Five micron-thick tissue sections were cut from these paraffin blocks. All of the bladder specimens were stained using Hematoxylin and Eosin ( $\mathrm{H}$ \& E) staining and Masson trichrome staining. Each section was observed under a light microscope at a magnification of x40-400.

The isometric contractile response was expressed as force (grams) per cross-sectional area (millimeter squared) of muscle $[6,8,9]$. The $\mathrm{EC}_{50}$ and $\mathrm{E}_{\max }$ values were obtained by a Macintosh computer (G3) loaded with Chart version 3.6.9 software and a Power Lab/16sp data acquisition system. Contractile data were calculated as grams of active 
force per cross-sectional area in square millimeter. The cross-sectional area was calculated by using the following equation: cross-sectional area = weight/ (length $\times 1.05$ ), where 1.05 is the assumed density of the muscle. A statistical comparison of differences between groups was performed with the use of analysis of variance and Fisher's multiple comparison tests. $\quad \mathrm{P}<0.05$ was regarded as the level of significance. 


\section{Results}

The rat bladder weight in the AUR group at two weeks was significantly larger than that of the controls, but the rat bladder weight in the AUR group at four weeks returned to the control level (Table 1).

In the voiding behavior studies, significant increase in micturition frequency per day and decrease in single voiding volume were observed at 3 days, and this voiding behavior was continued more than 2 weeks. However, no significant changes of urine output per day were observed in the experimental rats (Figure 1).

The cystometric studies showed a significant decrease in single-voided volume at 2 weeks rats compared to control or at 4 weeks rats. However, there were not statically significant differences in maximum detrusor pressure during voiding, single-voided volume, and residual urine volume between AUR groups and control group (Table 2).

The functional studies demonstrated that contractions to $100 \mathrm{mM} \mathrm{KCl}$, as well as $\mathrm{E}_{\max }$ and $\mathrm{EC}_{50}$ values to carbachol were not statically significantly different among the control group and the 2 weeks or 4 weeks AUR rat bladder smooth muscles (Table 3).

The histological studies showed significant infiltration of neutrophils and lymphocytes, and increase in turnover of epithelium at 2 weeks AUR rat bladder. In contrast, at 4 weeks AUR rats' bladder, it was observed a significant increase in fibrosis in submucosal layer, while infiltration of neutrophils and lymphocytes, and increase in turnover of epithelium were less evident. 


\section{Discussion}

Previously, we demonstrated that I-RI was caused by AUR and subsequent bladder catheterization, and it could be at least partly, responsible for the prolonged bladder dysfunction occurring after overdistension in the rat model [5]. In the same report, we reported that bladder dysfunction following catheterization was caused by reactive oxygen species (ROS) [5]. In order to investigate the effect of ROS on AUR-induced bladder dysfunction, we used a radical scavenger, edaravone, on the same rat model. We found that edaravone reduced the oxidative stress and prevented the bladder dysfunction caused by AUR and subsequent catheterization [6]. However, only limited information is available regarding the bladder function recovery after AUR. In the present study, we found significant increases in micturition frequency per day, which continued more than two weeks. Interestingly, in cystometric and organ bath studies, there were not statically significant differences in maximum detrusor pressure during voiding, residual urine volume, contractions to $100 \mathrm{mM} \mathrm{KCl}$, and $\mathrm{E}_{\max }$ and $\mathrm{EC}_{50}$ values to carbachol among the control group and 2 weeks or 4 weeks AUR rat groups. Kang et al. reported that by three days the ability of bladder to generate pressure returned completely to normal in a rat AUR model, and that AUR did not alter intravesical capacity as estimated by cystometory [10]. These data are in agreement with our cystometric and organ bath studies. Tammela et al. reported that AUR increased in bladder mass maximal at seven days, and that three weeks following AUR bladder functional state was not completely recovered [2]. They suggested that bladder overdistension has long-lasting effects on the bladder.

In this study, we demonstrated that a significant increase in micturition frequency per day and a decrease in single voiding volume were observed at 3 days, and this voiding 
behavior was continued more than two weeks. These data suggest that at least within two weeks, the experimental rats were under over active bladder (OAB) condition. Speakman et al. reported that in the porcine bladder outlet obstruction (BOO) induced an increase in sensitivity to exogenously applied agonists and a reduction in sensitivity to intramural nerve stimulation. Moreover, the authors noticed an inverse correlation between neuronal density and duration of obstruction in morphological studies [11]. They concluded that BOO was a cause of denervation supersensitivity. Steers et al. reported alterations in afferent pathways from the rat bladder in response to partial BOO [12]. In their report, they suggested that bladder afferents project to regions of the spinal cord known to regulate micturition and that these afferents can undergo morphological alterations and/or changes in axoplastic transport in response to urethral ligation. Seki et al. reported that the changes in the bladder smooth muscle membrane electrical properties induced by experimental $\mathrm{BOO}$ were only partially reversible following release of obstruction, and that the dysfunctional cystometric patterns associated with $\mathrm{BOO}$ might not only be due to changes in detrusor innervation but, fundamental reorganization of the detrusor's electrical syncytium with irreversible suppression of cell-to-cell transfer of electrical activity [13]. OAB is a symptom syndrome defined as "urgency, with or without urge incontinence, usually frequency and nocturia” [14]. OAB may be induced by BPH [15]. In the above studies the underlying pathophysiological mechanism of OAB induced by BOO may be explained as follows [2, 10-15]. BOO induces ischemia and/or hypoxia in the bladder, with subsequent partial denervation of the bladder. In this status, the partial denervation of the bladder induces supersensitivity to exogenously applied agonists such as acetylcholine, as well as reorganization of the detrusor's electrical syncytium with 
irreversible suppression of cell-to-cell transfer of electrical activity. Additionally, we demonstrated that AUR induced partial ischemia in the rat bladder, and that AUR associated bladder dysfunction is due to ROS caused by ischemia-reperfusion [5, 6]. As we have previously shown AUR and subsequent drainage in the bladder may induce partial denervation of the bladder.

In the histological studies, we observed significant infiltration of neutrophils and lymphocytes, and increase in turnover of epithelium 2 weeks after the induction of the AUR in the rat's bladder. These results were similar to histological changes in I-RI in the bladder reported in one previous report of our laboratory [16]. In that study, we observed that in I-R bladders there was heavy effluent of red blood cells from the rupture of microcirculation. Infiltration leukocytes were observed mainly in the submucosal area and smooth muscle layer.

In order to investigate $\mathrm{OAB}$ syndrome, adequate animal models are required. Moreover, the international literature provides several studies describing experimental models of $\mathrm{OAB}$ syndrome including pathologic conditions such as brain infarction, spinal cord injury, BOO and acetic acid or cyclophosphamide-induced cystitis model [17]. In this study, we demonstrated that AUR and subsequent bladder catheterization induced significant increases in voiding frequency at three days to more than two weeks after the induction of AUR in the rat model. We suggest this might be a useful model for OAB research, especially in non drug-induced rodent model.

In summary, we demonstrated that bladder dysfunction in a rat model caused by AUR needs more than two weeks of recovery period. The AUR-associated alterations in the bladder may represent a key clue to understand the pathogenesis of the OAB syndrome. 
The rat model of OAB may represent an adequate model for further investigation of the pharmaceutical management of OAB syndrome.

\section{Acknowledgements}

This study was supported by a grant in aid from the Ministry of Education, Science, and Culture of Japan (\#20591880). 


\section{References}

1 Choong S, Emberton E (2000) Acute urinary retention. BJU Int 85: 186-201.

2 Tammela TL, Levin RM, Monson FC, Wein AJ, Longhurst PA (1993) The influence of acute overdistension on rat bladder function and DNA synthesis. J Urol 150: $\underline{1533-1539 .}$

3 Gabella G, Uvelius B (1999) Structural changes in the rat bladder after acute outlet obstruction. Scand J Urol Nephrol 201: $\underline{32-37 .}$

4 Lin AT, Chen KK, Yang CH, Chang LS (2000) Mannitol facilitates rabbit urinary bladder recovery from overdistension injury. Urology 56: 702-707.

5 Saito M, Miyagawa I (2001) Bladder dysfunction after acute urinary retention in rats. J Urol 165:

6 Shimizu S, Saito M, Kinoshita Y, Kazuyama E, Tamamura M, Satoh I, Satoh K. Acute urinary retention and subsequent catheterization cause lipid peroxidation and oxidative DNA damage in the bladder: preventive effect of edaravone, a free-radical scavenger. BJU Int in press

7 Greenland JE, Brading AF (2001) The effect of bladder outflow obstruction on detrusor blood flow changes during the voiding cycle in conscious pigs. J Urol 165 : 
$\underline{245-248 .}$

8 Saito M, Kinoshita Y, Satoh I, Shinbori C, Suzuki H, Yamada M, Watanabe T, Satoh K (2007) Ability of cyclohexenonic long-chain fatty alcohol to reverse diabetes-induced cystopathy in the rat. Eur Urol 51: 479-488.

9 Saito M, Okada S, Kazuyama E, Satoh I, Kinoshita Y, Satoh K (2008) Pharmacological properties, functional alterations, and gene expression of muscarinic receptors in young and old type 2 Goto-Kakizaki diabetic rat bladders. J Urol 180: 2701-2705.

10 Kang J, Wein AJ, Levin RM (1992) Bladder functional recovery following acute overdistension. Neurourol Urodyn 11: $\underline{253-260}$.

11 Speakman MJ, Brading AF, Gilpin CJ, Dixon JS, Gilpin SA, Gosling JA (1987) Bladder outflow obstruction-a cause of denervation supersensitivity. J Urol 138: 1461-1466.

12 Steers WD, Ciambotti J, Etzel B, Erdman S, De Groat WC (1991) Alterations in afferent pathways from the urinary bladder of the rat in response to partial urethral obstruction. J Comp Neurol 310: 401-410.

13 Seki N, Karim OM, Mostwin JL (1992) The effect of experimental urethral obstruction and its reversal on changes in passive electrical properties of detrusor 
muscle. J Urol 148: 1957-1961.

14 Abrams P, Cardozo L, Fall M, Griffiths D, Rosier P, Ulmsten U, van Kerrebroeck P, Victor A, Wein A (2002) Standardisation Sub-committee of the International Continence Society. The standardisation of terminology of lower urinary tract function: report from the Standardisation Sub-committee of the International Continence Society. Neurourol Urodyn 21: 167-178.

15 Sexton CC, Coyne KS, Kopp ZS, Irwin DE, Milsom I, Aiyer LP, Tubaro A, Chapple CR, Wein AJ; EpiLUTS Team (2009) The overlap of storage, voiding and postmicturition symptoms and implications for treatment seeking in the USA, UK and Sweden: EpiLUTS. BJU Int 103 Suppl 3: 12-23.

16 Saito M, Wada K, Kamisaki Y, Miyagawa I (1998) Effect of ischemia reperfusion on contractile function of rat urinary bladder: Possible role of nitric oxide. Life Sci 62: PL149-PL156.

17 McMurray G, Casey JH, Naylor AM (2006) Animal models in urological disease and sexual dysfunction. Br J Pharmacol 147 Suppl 2: $\underline{\text { S62-S79. }}$ 


\section{Figure legends}

Figure 1. Micturition frequencies per day, single voided volume and urine out put per day in the experimental rats group (AUR group). *) significantly different from contols. $\mathrm{P}<0.05$ was regarded as the level of significance.

Figure 2. H\&E and Masson trichrome staining of control, 2 weeks and 4 weeks AUR rat bladders. $\mathrm{HE}$ and $\mathrm{MT}$ indicate $\mathrm{H} \& \mathrm{E}$ and Masson trichrome staining, respectively. Infiltrations of neutrophiles and lymphocytes, and increase in turnover of epithelium were observed at 2 weeks rat AUR bladder. Four weeks after the induction of AUR, a significant increase in fibrosis in submucosal layer was observed, whereas the infiltrations neutrophiles and lymphocytes as well as the increase in turnover of epithelium were less evident. Cont; bladder samples from control animals, 2 weeks; bladder samples 2 weeks after the induction of AUR. 4 weeks; bladder samples 4 weeks after the induction of AUR. 
Table 1. Bladder weight in the experimental rats

Bladder weight (mg)

\begin{tabular}{lc}
\hline Control $(\mathrm{n}=6)$ & $92 \pm 7$ \\
AUR at 2 weeks $(\mathrm{n}=6)$ & $153 \pm 22^{*}$ \\
AUR at 4 weeks $(\mathrm{n}=6)$ & $105 \pm 2$ \\
\hline *) significantly different from the contol group. $\mathrm{P}<0.05$ was regarded as the level of \\
significance.
\end{tabular}


Table 2. Cystometric studies in the experimental rats

\begin{tabular}{lccc}
\hline & Pdet $\left(\mathrm{cmH}_{2} \mathrm{O}\right)$ & single voided volume (ml) & residual urine (ml) \\
\hline Control & $29.7 \pm 4.7$ & $0.27 \pm 0.04$ & $0.012 \pm 0.013$ \\
AUR at 2 weeks & $28.6 \pm 2.2$ & $0.17 \pm 0.05^{*}$ & $0.025 \pm 0.025$ \\
AUR at 4 weeks & $33.9 \pm 7.7$ & $0.37 \pm 0.03$ & $0.035 \pm 0.021$ \\
\hline
\end{tabular}

Data are shown as mean \pm SEM of six separated determinations in each group. Pdet: maximum detrusor pressure during voiding. *) significantly different from the other groups. $\mathrm{P}<0.05$ was regarded as the level of significance. 
Table 3. $\mathrm{E}_{\max }$ and $\mathrm{EC}_{50}$ values for carbachol to bladder smooth muscle.

\begin{tabular}{lccc}
\hline & $\mathrm{E}_{\max }\left(\mathrm{g} / \mathrm{mm}^{2}\right)$ & $\mathrm{EC}_{50}\left(\mathrm{X} 10^{-6} \mathrm{M}\right)$ & $\mathrm{KCl}\left(\mathrm{g} / \mathrm{mm}^{2}\right)$ \\
\hline Control & $2.53 \pm 0.38$ & $1.5 \pm 0.4$ & $1.93 \pm 0.23$ \\
AUR 2 weeks & $2.33 \pm 0.32$ & $2.0 \pm 0.4$ & $1.45 \pm 0.21$ \\
AUR 4 weeks & $2.98 \pm 0.36$ & $1.4 \pm 0.1$ & $2.26 \pm 0.33$ \\
\hline
\end{tabular}

Data are reported as mean \pm SEM of six separated determinations in each group. $\underline{E}_{\text {max }}$ $\underline{\text { values; the maximal contraction produced by carbachol. EC }} \underline{50} \underline{\text { values; the concentration }}$ of carbachol that produces half-maximal contractile responses. $\mathrm{KCl}$ refers to contractile force to $100 \mathrm{mM} \mathrm{KCl}$. 

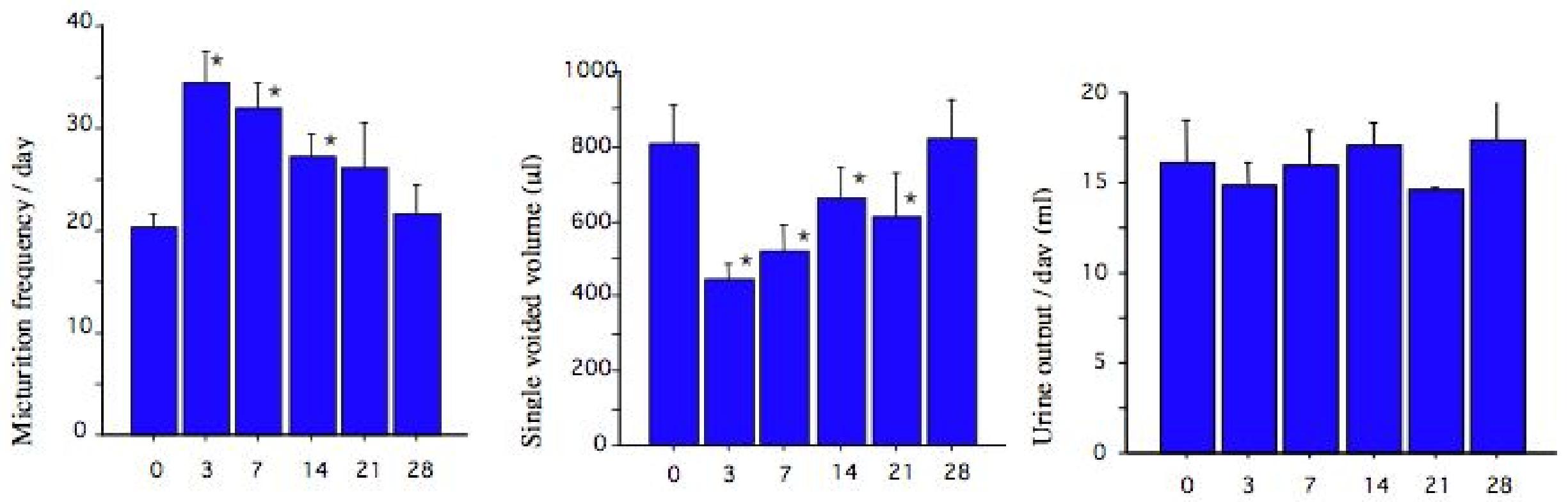


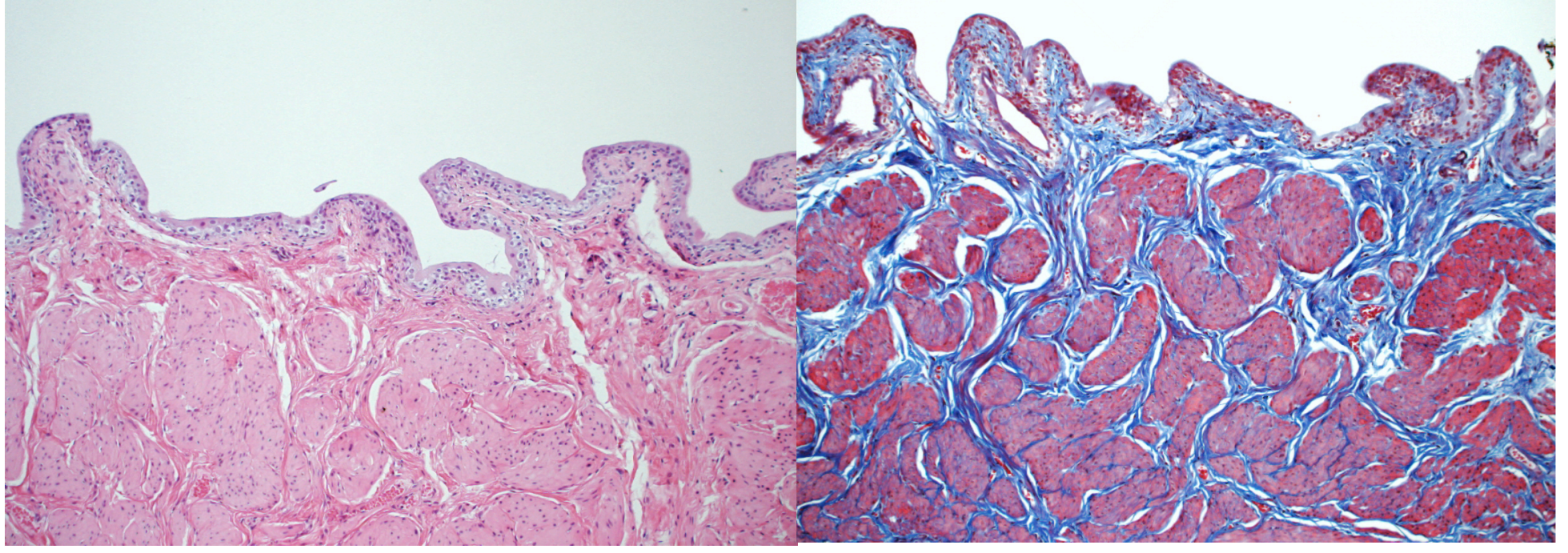

\section{Week HE}

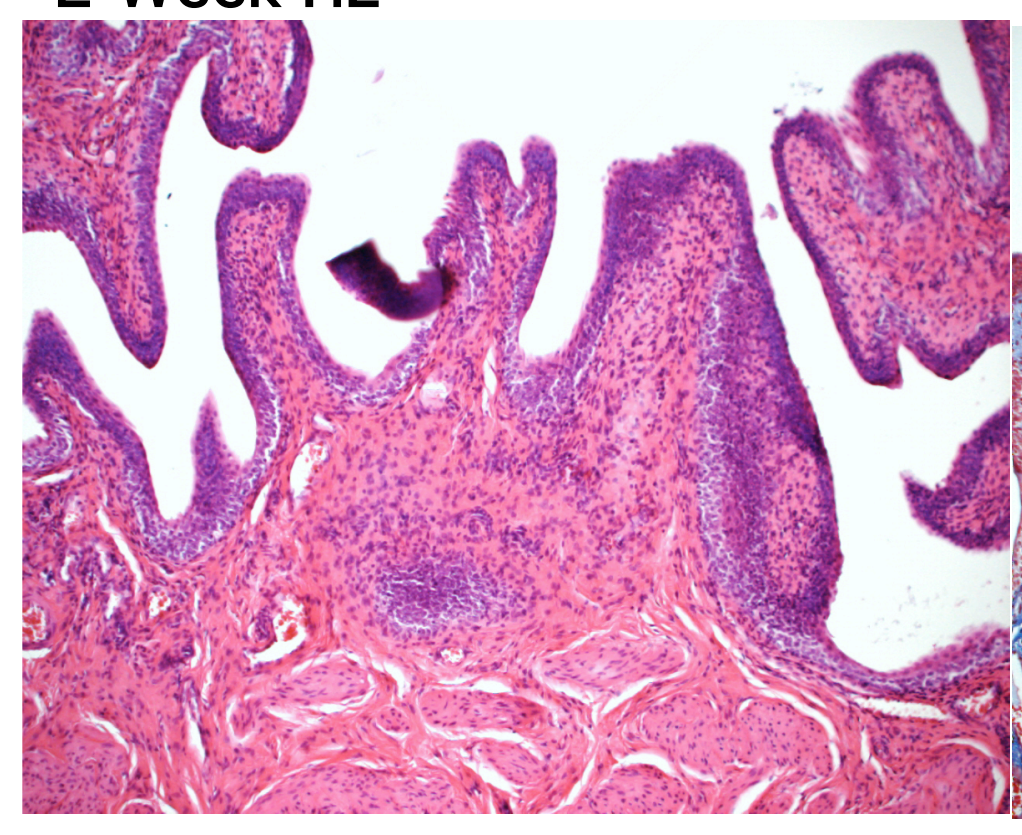

\section{Week HE}

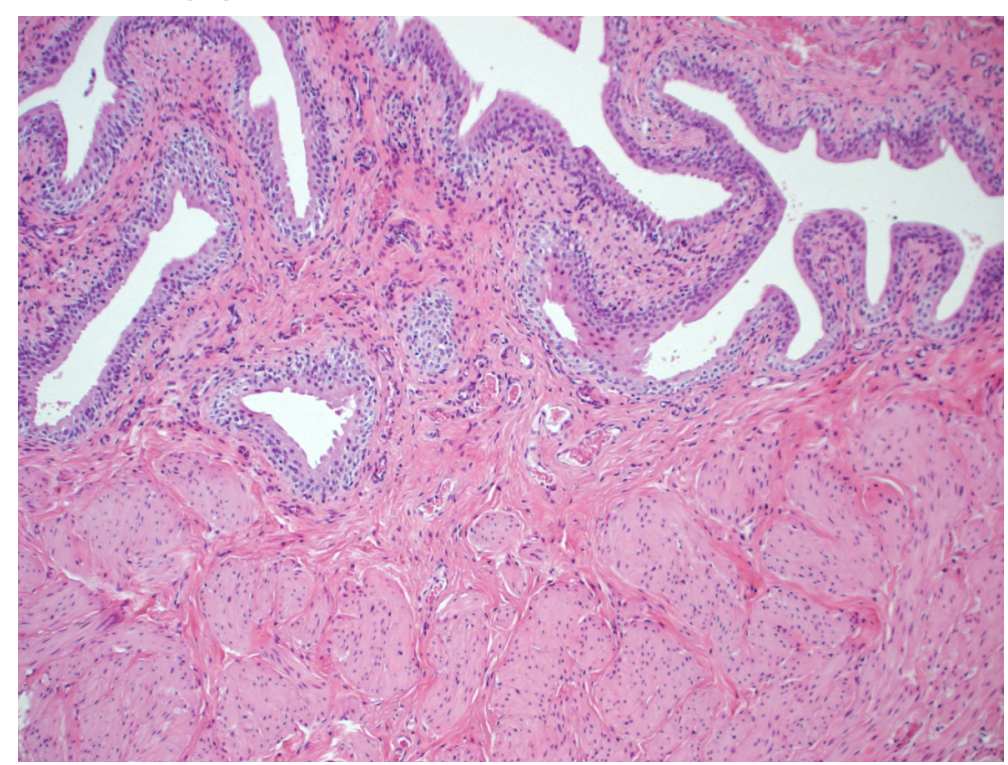

2 Week MT

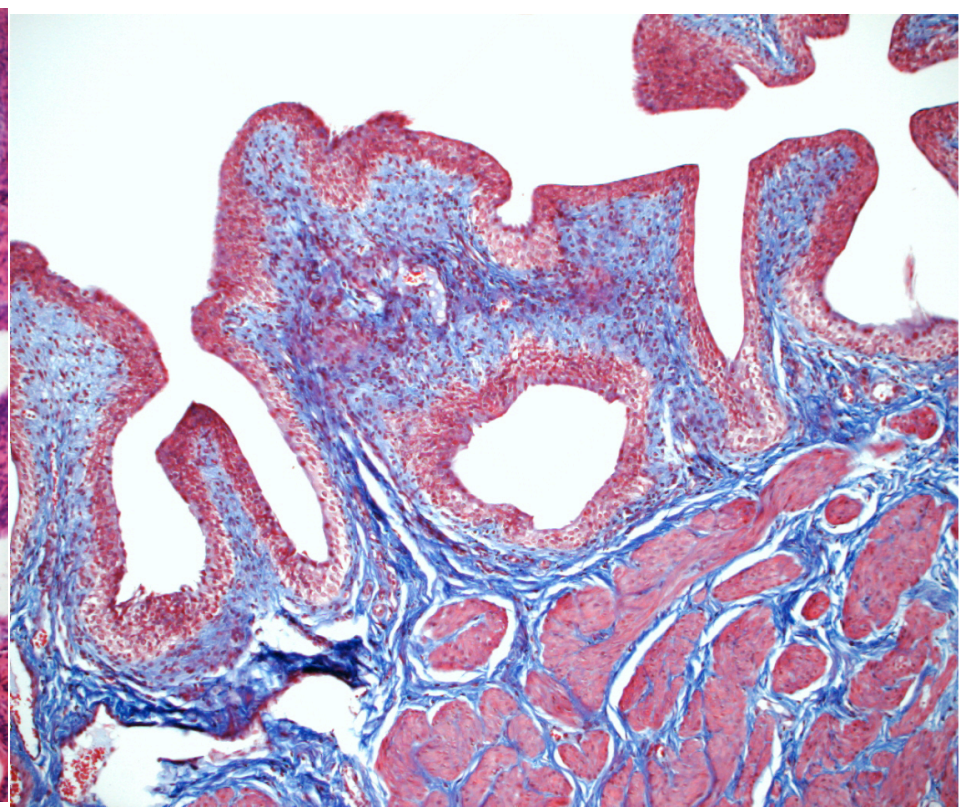

\section{Week MT}

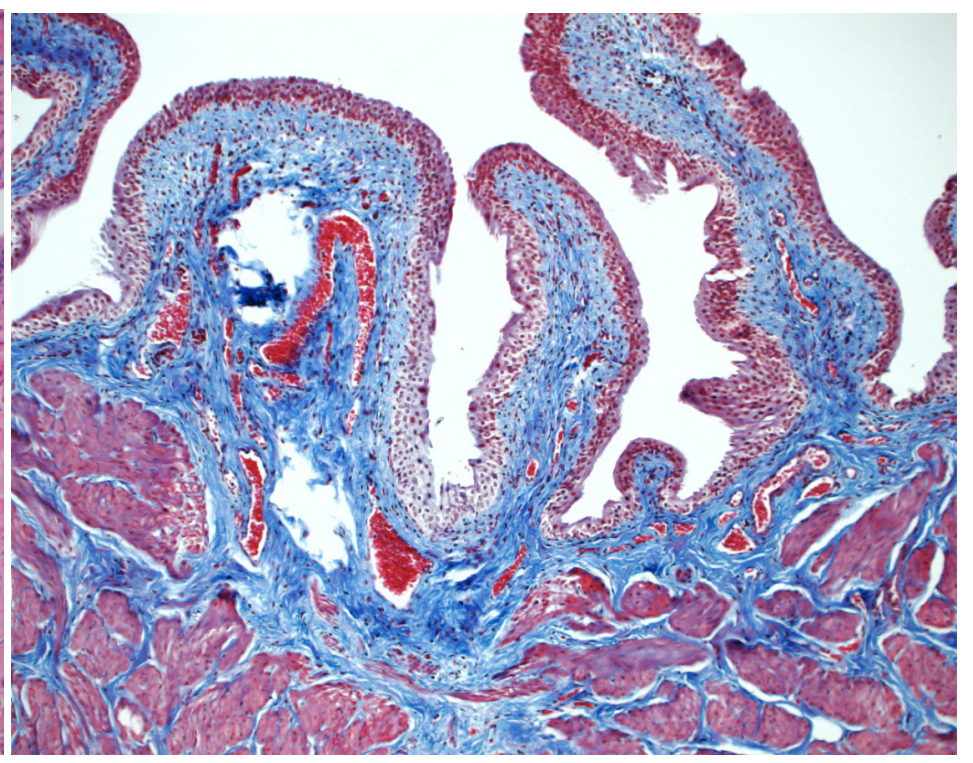

